\title{
COMPARISON OF ULTRASOUND WITH TENSILE TESTING OF THERMALLY DAMAGED POLYIMIDE INSULATED WIRING (MIL-W-81381)
}

\author{
Eric I. Madaras ${ }^{1}$ and Robert F. Anastasi ${ }^{2}$ \\ ${ }^{1}$ NESB, MS231, NASA Langley Research Center, Hampton, VA 23681 \\ ${ }^{2}$ U.S. Army Research Laboratory, Vehicle Technology Directorate, AMSRL-VT-S, NESB, \\ MS231, NASA Langley Research Center, Hampton, VA 23681
}

\begin{abstract}
Ultrasonic measurements were performed on MIL-W-81381/7, /12, and $/ 21$ wire, a polyimide (Kapton $\mathbb{B}$ ) insulated wire. The phase velocity for the 20-gauge MIL-W-81381/7 wire had a baseline value of $3023 \pm 78 \mathrm{~m} / \mathrm{s}$. After exposure to high temperatures, the wire's phase velocity rapidly increased, and reached an asymptotic value of $3598 \pm 20 \mathrm{~m} / \mathrm{s}$ after 100 hours exposure. Similar responses were measured in other gauges. The baseline measurements of Young's moduli resulted in values of $5636 \pm 486,7714 \pm 505$, and $8767 \pm 292 \mathrm{KSI}$ for the $20 \mathrm{ga}, 16 \mathrm{ga}$, and $12 \mathrm{ga}$. wires respectively.
\end{abstract}

\section{INTRODUCTION}

Electrical wiring is subjected to heat, cold, moisture, strain and vibrations, which eventually cause damage to the wire insulation and can lead to failure of the wire conductor. In most cases these environmental and operational conditions are modest and wiring is used safely for years, but in the case of aerospace and aeronautics usage, these conditions are more extreme and can cause the insulation to chafe, to become brittle and to crack prematurely, sometimes with serious consequences. The common practice to deal with wiring faults is often after-the-fact, usually done in response to an instrument or system failure. In those cases, wire inspections are done visually to find the obvious cracks, damage, and burns. However, visual inspections give little quantitative information about the condition of the wire-insulation prior to wire failure or the condition of visually intact wire which is near critical failure.

To investigate the concept of using guided waves for insulation assessment, a series of experiments were conducted. Early reports on ultrasonic testing of wiring have suggested the efficacy of this concept as it is applied to electrical wiring [1,2]. The first test was to 
evaluate consistency of theory and a simple model consisting of a solid cylinder and then a solid cylinder coated with a polymer. Several guided modes were generated and their character were compared with modeling results. Next, a series of aviation grade wiring was tested to evaluate their ultrasonic properties. The wiring, designated as MIL-W-81381 wiring, is insulated with polyimide layers. This wiring is commonly referred to as Kapton ${ }^{\circledR}$ wiring which has been identified as an aging wiring that can fail prematurely under certain conditions. The conductors for these materials have a stranded conductor construction rather than the solid cylindrical form of our initial model and the insulation can be wrapped rather than a continuous cylinder, and thus it is important to evaluate the character of the ultrasound signal to see if it also followed the simple model or if a new model would be necessary. These wires were exposed to high temperatures for various times to create wiring that exhibited various conditions. Subsequent to ultrasonic testing, several subsets of these wires were stressed in a small table top load frame to estimate their Young's modulus and then to compare those values with the ultrasonically measured values.

\section{THEORY}

In its basic geometry, the insulated wire may be considered a cylindrical clad waveguide. A number of researchers have examined acoustic guided wave propagation in cylindrical geometries and the reader is referred to those manuscripts for detailed equations [3-6]. Some applications of ultrasonic guided waves include material testing or characterization of wire and fibers and their use as ultrasonic delay lines [7, 8].

In general many acoustic wave modes will propagate in an isotropic cylinder. The lowest order modes of vibration are the axial symmetric modes, which can be divided into axialradial and torsional modes. The next order mode of vibration is the flexural mode and higher modes are screw modes [4]. Figure 1a shows the lowest branch of the axial-radial mode extending to zero frequency where the limiting phase velocity is called the bar velocity in the simple isotropic case, and is related to the Young's modulus of the material. In the low frequency regime this mode is nearly non-dispersive. As the frequency is increased the phase velocity drops and then asymptotically approaches the Rayleigh velocity at higher fre-
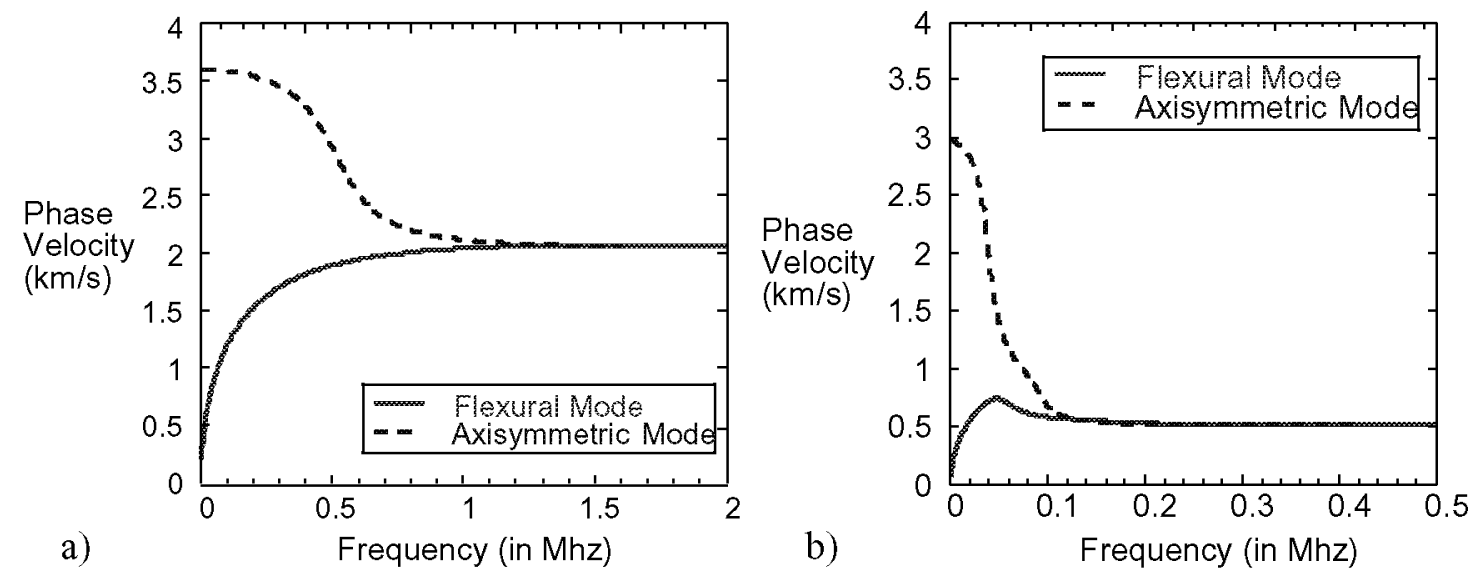

Figure 1. a) The phase velocity of the first axisymmetric wave mode and the first flexural wave mode of a solid $1 / 8$ " brass cylinder. b) The phase velocity of the first axisymmetric wave mode and the first flexural wave mode of a solid $1 / 8$ " brass cylinder with a layer of insulation. 
quencies. Figure 1a also shows the first flexural mode's dispersion behavior which is characterized by a zero phase speed at zero frequency. As the frequency increases, the phase velocity increases. It asymptotically approaches the axisymmetric wave speed from below.

The addition of a layer of polymer insulation has a significant effect on the two low order modes as seen in Figure 1b. The effect is to lower the frequency range where dispersion is most noticeable and to lower the phase velocity. The low frequency axisymmetric wave velocity drops about $20 \%$ in this instance and the high frequency asymptotic limit is related to the plastic's properties rather than the brass' properties.

\section{EXPERIMENT}

The ultrasonic experimental system consisted of a pitch catch mode system. An ultrasonic spike generation pulser generated ultrasonic waves. Two similar longitudinal broad band transducers were used for generation and reception. The transducers had a bandwidth of $50 \mathrm{KHz}$ to $2 \mathrm{MHz}$. On reception, a $20 \mathrm{KHz}$ to $2 \mathrm{MHz}$ pre-amplifier with 40 or $60 \mathrm{~dB}$ of switchable gain fed into an amplifier with a maximum of $42 \mathrm{~dB}$ of gain and with the bandwidth set at $10 \mathrm{KHz}$ to $300 \mathrm{KHz}$. The output of the amplifier was recorded by an 8-bit/500 $\mathrm{MHz}$ digitizing oscilloscope. That signal was averaged 1000 times. Two hundred $\mu$ secs of signal were recorded. The transducers were mounted in simple sample holders that held the wire across the center of the transducer's face. The spacing of the transducers was adjusted from $3 \mathrm{~cm}$ to $29 \mathrm{~cm}$ to measure a range of arrival times and spacings. The wire samples were nominally $60 \mathrm{~cm}$ long. For the case of measuring the phase velocity, the first arrival peak of the signal at each distance was used to estimate a velocity. A least squares routine was used to calculate the velocity and to estimate the error of the velocity from the distance versus time data.

To generate thermal damage in wiring, sets of 12 wires for each gauge and time at temperature were placed in a large laboratory oven and heated to $370^{\circ} \mathrm{C}$. The oven was able to reach the dwell temperature in about an hour. The cool down period lasted considerably longer. The wires were removed when the temperature fell below at least $200^{\circ} \mathrm{C}$. For the wires that were tested, sets of each wire were heated to their dwell temperature for $0,3,6,9$, $12,1520,30,40$, or 100 hours. These wires were subsequently ultrasonically measured.

The Young's moduli were measured using a small computer controlled, electromechanically actuated table top load frame with a 1000 pound capacity load cell. An extensometer with a 1" gauge length was used for the displacement measurements. During tensioning of the wire samples, at the first detectable signs of yielding, the loading was halted to keep the measurements in the linear stress-strain regime. The resulting load levels were very small because of the small size of the wires. Typically, the range was from 20 to 100 pounds. Four locations were measured in the central test region of each wire, well away from the load frame grips, to average the wire's variability. Efforts were made to try to make measurements that avoided locations with evidence of residual stress. This was accomplished by excluding locations which exhibited non-linear stress-strain curves and locations where the Young's modulus results were significantly different $(>20 \%)$ on opposite sides of the wire. This method of measuring the Young's modulus represents contributions from both the wire core and the insulation combined. 


\section{RESULTS}

\section{Solid Insulated Wire Model.}

Initial measurements were carried out on a simple model of an insulated wire to identify various wave modes. This model consisted of a solid, $3.23 \mathrm{~mm}$ diameter aluminum rod with and without a polymer coating. The polymer coating simulating the wire insulation, a polyolefin, is a thermoplastic heat-shrink material that had a thickness of $0.57 \mathrm{~mm}$. Published density and modulus of this material is $0.971 \mathrm{gm} / \mathrm{cm}^{3}$ and $1.2 \mathrm{GPa}$ respectively, and the measured longitudinal wave velocity is $1870 \mathrm{~m} / \mathrm{s}$. [9] The final diameter of the model was $4.37 \mathrm{~mm}$. The published aluminum properties are $70.76 \mathrm{GPa}$ and $2.7 \mathrm{gm} / \mathrm{cm}^{3}$ for young's modulus and density. [10] They predict a bar velocity of $5119 \mathrm{~m} / \mathrm{s}$ for a bare aluminum rod.

Typical ultrasonic signals in the bare aluminum rod and in the insulated aluminum rod are shown in Figures $2 a$ and $2 b$ respectively. In Figure $2 a$ there is an initial small signal at about $30 \mu \mathrm{s}$ and then a much larger signal starts near $50 \mu \mathrm{s}$. In Figure $2 \mathrm{~b}$, the initial signal starts at about $35 \mu \mathrm{s}$ and then a much larger signal starts about $60 \mu \mathrm{s}$. In both figures, these signals are followed by additional signals that are either additional modes or reflections. Those signals overlap sufficiently to make identification difficult. In both figures, the second arriving mode clearly illustrates dispersive behavior with the higher frequencies traveling faster. Also, it can be noted that the bare aluminum rod has a much higher frequency content. The phase velocity of the axisymmetric mode of the bare rod and the polymer coated aluminum rod were measured to be $5119 \pm 26 \mathrm{~m} / \mathrm{s}$ and $4597 \pm 36 \mathrm{~m} / \mathrm{s}$ respectively. The frequencies of Figure $2 \mathrm{a}$ has a range of approximately 50 to $240 \mathrm{KHz}$ while the fre-

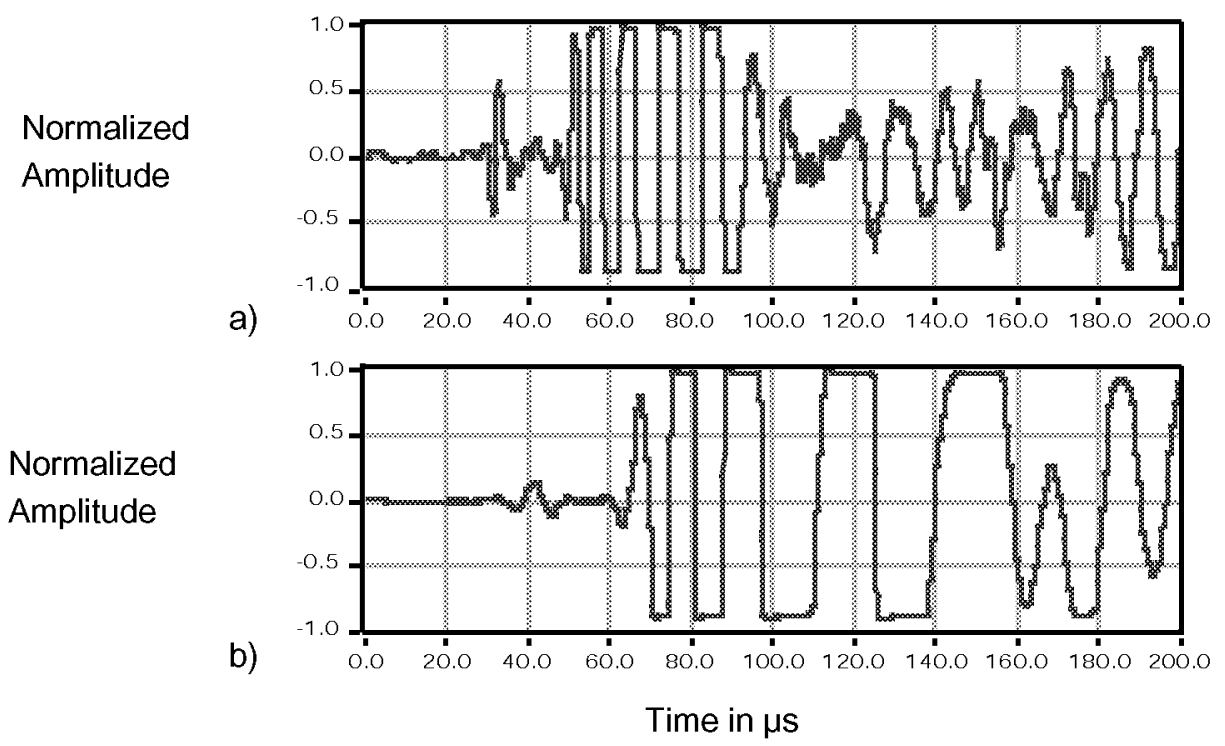

Figure 2. Typical ultrasonic signals. a) The signal generated and recorded from a $1 / 8$ " aluminum rod. The first arrival at $30 \mu \mathrm{s}$ is the axisymmetric wave, followed by the flexural mode at about $50 \mu \mathrm{s}$. b) The signal generated and recorded from a $1 / 8$ " aluminum rod with a polymer coating. The first arrival at $35 \mu$ s is the axisymmetric wave, followed by the flexural mode at about $60 \mu \mathrm{s}$. The dispersion of the flexural mode is evident in both reference signals. 
quencies of Figure $2 \mathrm{~b}$ has a range of approximately 20 to $100 \mathrm{KHz}$.

\section{MIL-W-81381 Wire, Polyimide Insulation.}

To see if this method might be applied to aging wiring, several mil-spec wire samples were heat damaged to induce changes in the insulation. Sets of twelve wires of type MIL-W81381 were heated to $370^{\circ} \mathrm{C}$ and held at that temperature for different dwell times and subsequently measured. This wiring had a listed maximum safe operating temperature of $200^{\circ} \mathrm{C}$. In addition, a set of twelve wires that were not exposed to thermal degradation were used for baseline measurements. Visually, the wires darkened slightly with time in the oven, but did not display overt damage. Figure 3 shows the resulting ultrasonic data. The phase velocity for the 20 -gauge MIL-W-81381/7 wire had a baseline value of $3023 \pm 78 \mathrm{~m} / \mathrm{s}$. After exposure to the high temperatures, the wire's phase velocity rapidly increased, and reached an asymptotic value of $3598 \pm 20 \mathrm{~m} / \mathrm{s}$ after 100 hours exposure. Similar behavior was measured for the 16 gauge MIL-W-81381/21 wire and 12 gauge MIL-W-81381/12 wire which had baseline values of $3225 \pm 22 \mathrm{~m} / \mathrm{s}$ and $3403 \pm 33 \mathrm{~m} / \mathrm{s}$ respectively, and reached asymptotic values of $3668 \pm 19 \mathrm{~m} / \mathrm{s}$, and $3679 \pm 42 \mathrm{~m} / \mathrm{s}$ respectively.

\section{Young's Modulus Results.}

Some preliminary results for Young's modulus measurements in MIL-W-81381 wires are shown in Figure 4a. The Young's moduli for the baseline wires that were tested had resulting values of $5636 \pm 486,7714 \pm 505$, and $8767 \pm 292 \mathrm{KSI}$ for sets of six wires from the ultrasonically measured $20 \mathrm{ga}, 16 \mathrm{ga}$, and $12 \mathrm{ga}$. wires respectively. In addition, measurements on the $12 \mathrm{ga}$. wires indicated that the moduli were increasing at early times of thermal damage with values of $9363 \pm 716 \mathrm{KSI}$ at zero hours of dwell time at temperature, $10248 \pm 374 \mathrm{KSI}$ at 3 hours, and $9456 \pm 390$ at 20 hours. Table 1 provides dimensional

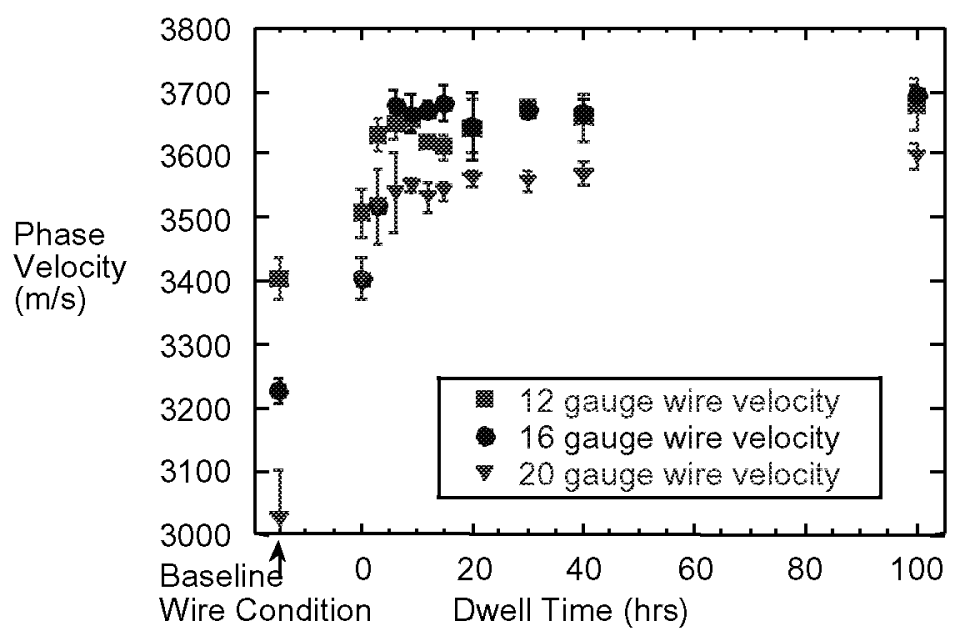

Figure 3. Ultrasonic axisymmetric phase velocity measurements on 12 (2), 16 ), and 20 (w) gauge polyimide insulated wire (MIL-W-81381). 
Table 1: General information about Mil-W-81381 wiring

\begin{tabular}{|c|c|c|c|}
\hline Gauge & 12 & 16 & 20 \\
\hline Mil-W-81381 spec. Variant & $/ 12$ & $/ 21$ & $/ 20$ \\
\hline Overall Diameter (mm) & 2.50 & 1.63 & 1.29 \\
\hline Wire Bundle Diam. (mm) & 2.09 & 1.33 & 0.94 \\
\hline Insulation Thickness (mm) & 0.21 & 0.15 & 0.17 \\
\hline Wire strands per Bundle & 37 & 19 & 19 \\
\hline Wire Strand Gauge & 28 & 29 & 32 \\
\hline Conductor Type & $\begin{array}{l}\text { Ni coated } \\
\text { copper }\end{array}$ & $\begin{array}{l}\text { Sn coated } \\
\text { copper }\end{array}$ & $\begin{array}{l}\text { Ag coated } \\
\text { copper }\end{array}$ \\
\hline Max. operating Temp. & $200^{\circ} \mathrm{C}$ & $200^{\circ} \mathrm{C}$ & $200^{\circ} \mathrm{C}$ \\
\hline
\end{tabular}

information about the wires. The variations in the Young's modulus are in part a reflection the relative ratios of the wire core cross sectional area to the insulation cross sectional are

\section{DISCUSSION}

Figure 1 in the theory section predicts some general properties for guided waves in clad and unclad cylinders. In the measurements that were made, the results correspond to those predictions. As seen in Figure 2, the axisymmetric wave arrives first and doesn't appear very dispersive, which is predicted for low frequencies. The flexural mode arrives next, and displays a significant dispersive character with the lower frequencies traveling slower as predicted by the theory. In addition, the dispersive character shifts to lower frequencies for the clad rod because of the thicker diameter of the rod and insulation, which is also evident from Figure 1. The axisymmetric mode's velocity measurement in the unclad
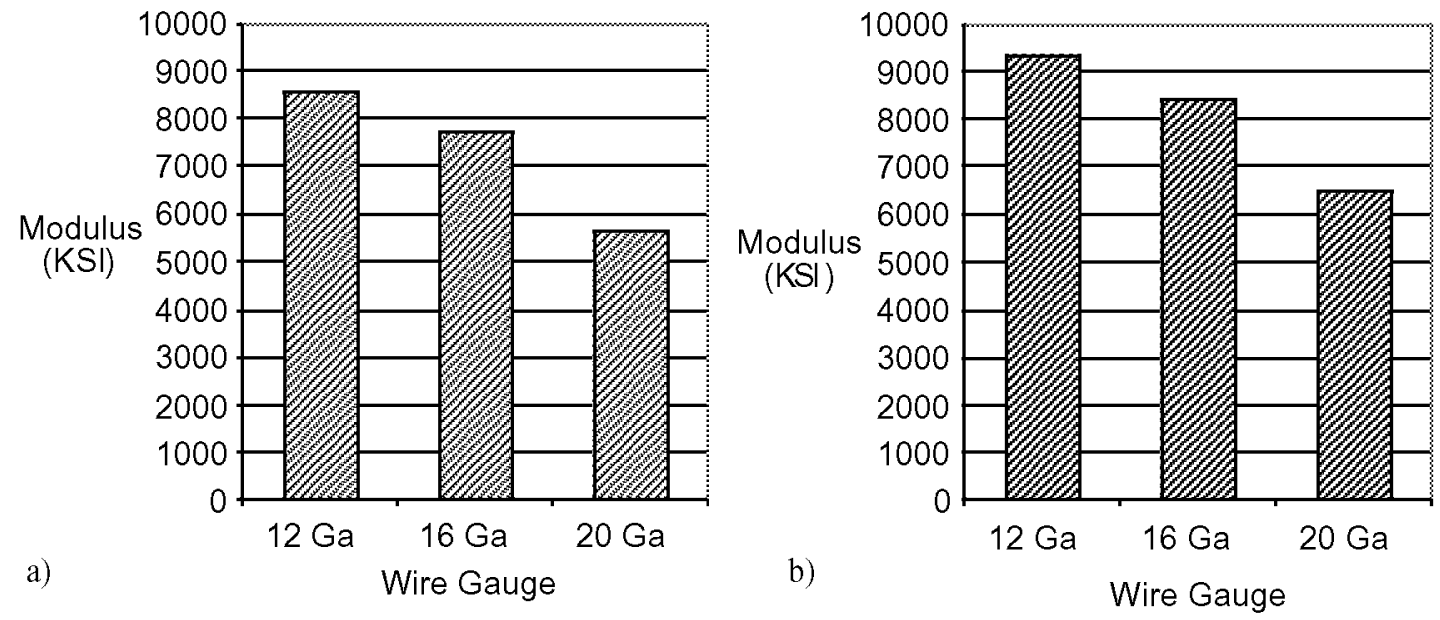

Figure 4. Modulus measurements. a) The Young's moduli measured for Kapton wiring, gauges 12, 16, and 20. b) Estimates of the Young's moduli calculated from the ultrasonic data for gauges 12, 16, and 20. 
aluminum rod was within $0.2 \%$ of the calculated value base on published Young's modulus and density values for aluminum. Finally, the velocity of the axisymmetric wave and the flexural wave are significantly lower when comparing the low frequency unclad rod with the clad rod, which is evident in the waveforms in Figure 2. In the case of the unclad aluminum rod, measurements for the axisymmetric wave speed were $5119 \mathrm{~m} / \mathrm{s}$ while for the clad aluminum rod, measurements of the axisymmetric wave speed were $4597 \mathrm{~m} / \mathrm{s}$, a $10 \%$ decrease. The difference in the measured values between the clad and unclad examples are similar to the qualitative behavior predicted in Figure 1. An important conclusion is that some of the ultrasonic energy is traveling in the insulation and can thus be used to evaluate the wire insulation condition. The relative signal strengths between the axisymmetric wave and the flexural wave is believed to result from the manner in which the ultrasound is generated.

Since naturally aged wiring with a well characterized history is not available at this time, high heat was used to try to produce a range of material properties in a rather short time frame. Thermal "aging" is viewed as a way to make quick changes in material properties of wiring with some relevancy. Qualitatively, the measured ultrasonic rf waveforms for all the wiring materials used in this study were similar to those ultrasonic rf waveforms seen for the polymer clad solid rod, suggesting the applicability of the model to wiring despite the more complicated geometry of wire construction. Thus, it appears that ultrasonic transmission down concentric cylinders with intimate contact is a reasonably good model for the insulated wire, even with a stranded conductor and wrapped insulation.

It was anticipated that the velocity behavior of the MIL-W-81381 wire to heat damage would be to increase as the insulation was thermally damaged. This was based on the assumption that the insulation would harden with thermal damage. Our choice of temperature with which to damage the wires was based mostly on the perceived visual changes that were induced. It was expected that the velocity would gradually increase. The general pattern was for the velocity to increase monotonically, except the velocity changes seemed to occur at very early times rather than slowly over longer periods.

The load frame measured values of Young's modulus show a strong correlation with the ultrasonically measured data. In Figure 4, in addition to the measurements of the Young's moduli using a load frame, we also show the prediction of the Young's moduli based on the low frequency ultrasonic measurements. It was anticipated that the axisymmetric wave speed derived Youngs' moduli would be similar to the load frame determined moduli. What is seen is that both sets of data show a correspondence between the two methods. They are not identical, but rather the ultrasonically estimated Young's modulus are consistently higher by about 10\%. A similar affect was found for the 12 gauge wire measurements of Young's modulus made with the load frame on wires with different thermal damage times. When compared with calculations based on the ultrasonic data, that data also showed a systematic offset. It is possible that in the effort to exclude residual stress effects, the load frame measurement of Young's modulus might have been biased. This hypothesis will be investigated in the future.

\section{CONCLUSION}

This work demonstrated the generation of ultrasonic axisymmetric and flexural guided waves in a clad and unclad aluminum solid rod and in insulated wire samples. Clip-on 
piezoelectric transducers were used to generate and detect ultrasonic guided cylindrical waves. A qualitative relationship with theory for cylindrical wave guides was demonstrated from the guided wave measurements on the unclad and clad aluminum rod. A qualitative similarity between our model and aviation grade wiring was seen. The axisymmetric wave mode was then measured for numerous samples of aviation wiring that had been subjected to extreme temperatures to help induce material changes in short time spans. The effects of thermal degradation were detected by measuring the guided wave speed in the MIL-W81381 wiring in all gauges measured. In addition, a simple correlation was seen between the ultrasonic data and measurements of the wire's Young's modulus.

\section{REFERENCES}

1. Anastasi, R. F., and Madaras, E. I., "Ultrasonic Guided Waves For Aging Wire Insulation Assessment," in 28th Annual Review Of Progress In QNDE, Eds. D. O. Thompson and D. E. Chimenti, AIP, NY, 21a, 255-261, 2002,

2. Madaras, E. I., and Anastasi, R. F., "Investigating The Use Of Ultrasound For Evaluating Aging Wiring Insulation," Fifth Joint NASA/FAA/DoD Aging Aircraft Conference, 2001, 3 Meeker, T. R., and Meitzler, A. H., 1969, "Guided Wave Propagation In Elongated Cylinders and Plates," In Physical Acoustics - Principles and Methods, Ed. W. P. Mason, Academic Press, NY, 1a, 111-167.

4. Thurston, R. N., J. Acoust. Soc. Am., 64, 1, 1-37, (1978)

5. Mcniven, H. D., Sackman, J. L., and Shah, A. H., J. Acoust. Soc. Am., 35, 10, 1602$1609,(1963)$.

6. Abramson, H. N., J. Acoust. Soc. Am., 29, 1, 42-46, (1957).

7. Madaras, E. I., Kohl, T. W., and Rogers, W. P., "Material Property Characterization and Pulse Propagation In Thin Drawn Wire Waveguides," in IEEE Ultrasonics Symposium, Ed. B.R. McAvoy, IEEE, NY, 957-962 (1992).

8. Madaras, E. I., Kohl, T. W., and Rogers, W. P, J. Acoust. Soc. Am., 97, 1, 252-261, (1995).

9. Gangal, S. V., and Grot, W., "Tetrafluoroetheylene Polymers," in Encyclopedia Of Polymer Science and Engineering, Eds. H. F. Mark and J. I. Kroschwitz, Wiley, N.Y., 16, 577-648, (1989).

10. Electronic Source: The online materials information resource, "http:// www.matweb.com/specificmaterial.asp?bassnum $=04306 \&$ group $=$ general", (Accessed July $5,2000)$ 